www.mdpi.com/journal/sensors

Conference Proceedings Paper - Sensors and Applications

\title{
A Low Cost Magnetic Resonance Relaxometry Sensor
}

\section{Elizabeth R. Dye ${ }^{1}$, Theodore Hughes-Riley ${ }^{1}$, Steven T. Parslow ${ }^{1}$, Michael I, Newton ${ }^{1}$ and Robert H. Morris ${ }^{1 \text {,* }}$}

1 School of Science and Technology. Nottingham Trent University, United Kingdom: elizabeth.dye2013@my.ntu.ac.uk; theodore.hughesriley@ntu.ac.uk; steven.parslow2008@my.ntu.ac.uk; michael.newton@ntu.ac.uk

* Author to whom correspondence should be addressed; E-Mail: rob.morris@ntu.ac.uk; Tel.: +(44) 01158483315.

Published: 10 November 2015

\begin{abstract}
Magnetic resonance relaxometry, conducted by measuring relaxation parameters at different field strengths, has become an increasingly popular technique in recent years. This technique, known as field cycling, often uses expensive and large electromagnets. In this work we present a small, portable field cycling sensor. Fast field cycling is a technique that uses a varying magnetic field applied to a sample, polarising it at a high field, allowing it time to develop at a lower field and then collecting the data at the same initial high field. This causes changes in $T_{1}$ and can reveal interesting properties of the samples not seen by traditional methods. A prototype portable magnetic resonance sensor that undertakes relaxometry measurements using fast field cycling has been developed using a combination of permanent magnets which has been used to conduct preliminary studies on a water sample. We demonstrate the effectiveness of this sensor by conducting measurements of $\mathrm{T}_{1}$ at different field strengths.
\end{abstract}

Keywords: Magnetic resonance; Relaxometry; Fast Field Cycling; Desktop probe; Low cost

\section{Introduction}

Magnetic resonance (MR) has found countless applications since its discovery, it's most popular being medical imaging. It allows for the visualization of the internal structure of a sample without the need to destroy it. Despite this ability, traditional MRI machines are limited by their size and cost, meaning many institutions cannot afford to buy and maintain an MRI scanner. Additionally, their size 
means that it is impractical to take them into the field where many interesting experiments can be conducted. Their inner diameter (often referred to as the bore diameter) typically requires samples to be collected from their environment in order to perform laboratory testing [1].

Taking measurements of relaxation parameters is an alternative to imaging as is used in traditional MRI. It requires much smaller apparatus and is able to operate at lower field strengths and is therefore generally much cheaper. Due to this, MR sensors of this type have the application of being taken into the field to record data in situ [1].

MR probes the physical properties of water non-invasively. It does this through the use of a static magnetic field, a radio frequency (RF) coil and electronics which generate a series of appropriate RF pulses, commonly referred to as a pulse sequence. The protons within the water align within the magnetic field and through the application of a series of appropriate radio frequency pulses, the spinspin lattice relaxation time $\left(T_{1}\right)$ of a sample can be acquired which gives indication to the properties of the water molecule's environment. The frequency of the pulses is dependent on the magnetic field within the sensor, as is the $T_{1}$. This dependence of $T_{1}$ is not linear which can often reveal interesting features of the system [2]. An experiment known as field cycling can be used to determine the dependence of $T_{1}$ on the field strength and has found numerous applications in various fields such as investigating protein solutions, cells, tissues and liquid crystals [3].

In order to collect $\mathrm{T}_{1}$ data for a variety of different magnetic field strengths, a large number of probes or broadband RF coils would be required, both of which are very challenging and expensive to construct. This has led to the widespread adoption of the fast field cycling technique. Fast field cycling NMR relaxometry allows the magnetic field that the sample experiences to be varied whilst excitation and detection always occur at the same field. Relaxation times $\left(\mathrm{T}_{1}\right)$ can be taken for the sample at lower field strength than the detection field without the need to retune the radio frequency coil (RF coil). This allows measurements to be taken in quick succession without need of human intervention [3].

At very low field strengths (16-69mT), quadrupole dips can be found in samples with multiple species where the proton resonating frequency crosses with the nuclear quadrupole resonance (NQR) frequencies. These can be used to identify the bonding, structure and even temperature of the sample [4].

Field cycling apparatus that uses superconducting magnets dominate the market at current and these systems are very expensive to acquire and maintain as they require cryogenic cooling to function. This work shows a low-cost permanent magnet system which is a possible alternative to commercial superconducting magnet systems.

\section{Experimental Section}

The sensor is composed of two separate magnet arrangements: A Halbach design (Fig.1 a), which is responsible for transmitting RF to and collecting the signal from the sample, and a perpendicular magnet arrangement (Fig. 1 b), which is responsible for the varying magnetic field applied by virtue of moving the sample physically. The magnets are arranged with respect to each other as shown in Figure 1. This results in a $U$ shaped magnetic field that is experienced by a sample moving out of the Halbach 
and through the perpendicular magnet arrangement. The right hand, increasing side of this $U$ shape is used to vary the evolution field strength.

Figure 1.3D rendering of the magnet arrangement.

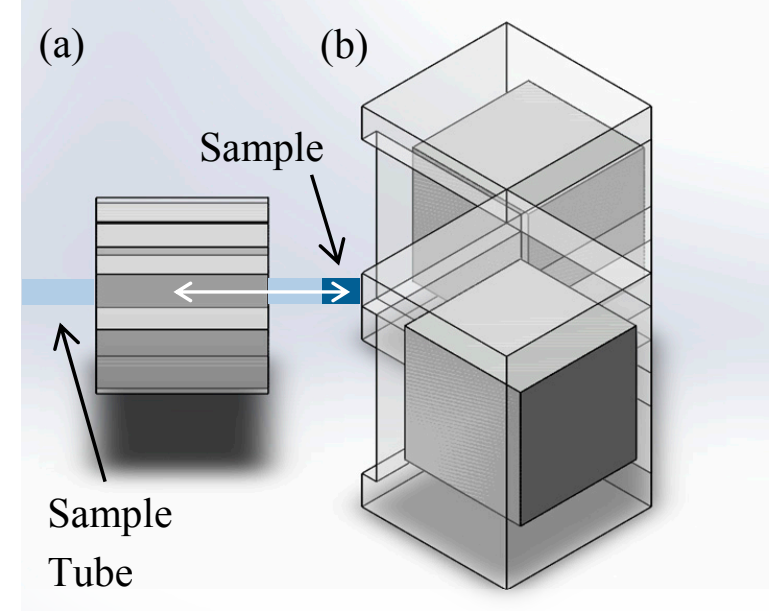

In the Halbach configuration, 8 N42 neodymium magnets (42 x $8 \times 10 \mathrm{~mm}^{3}$, first4magnets, UK) were arranged to be $20 \mathrm{~mm}$ from the magnet opposite in the octagonal Halbach array, these were placed in an acrylic housing that ran the length of the magnets. The bore diameter was $15 \mathrm{~mm}$ and ran through the entire length of the housing. This generated a magnetic field within the centre of $490 \mathrm{mT}$ at $293 \mathrm{~K}$.

An RF coil resides within the centre of the Halbach magnet arrangement which takes form of a 12turn copper solenoid with a $13 \mathrm{~mm}$ diameter, this is connected to a parallel-series tuning board with two variable capacitors completing the circuit which resonated at $20.86 \mathrm{MHz}$.

In the perpendicular magnet configuration, two magnets (40 x 40 × $20 \mathrm{~mm}^{3}$, HKCM, Germany) were mounted to form a right angle with an inside spacing of $15 \mathrm{~mm}$. The range of magnetic field strengths were from 40 to $420 \mathrm{mT}$.

A simulation (Fig.2) was conducted in FEMM version 4.2 [5]. The results demonstrate that a design such as this should be well suited to fast field cycling. The variation in the field as the sample passes between the magnets presents a gradient no higher than would be typically experienced in a traditional system.

A length of pipe of inner diameter $4 \mathrm{~mm}$ passes through the RF coil of the Halbach and through the perpendicular magnet as shown in figure 1. The sample is bound on both sides by air to ensure signal is only measured from water that has experienced the relevant field. The sample was moved between the areas of different field strength virtue of a custom built syringe pump connected to the end of the tube. By infusing and withdrawing the syringe pump, the sample is inverted in the Halbach, pushed into a new field for evolution and returned to the coil for signal detection.

$\mathrm{T}_{1}$ was measured using a fast field cycling modified inversion recovery pulse sequence [6,7] (Fig.3) implemented through a Kea 2 spectrometer (Magritek, New Zealand) running Prospa 3.22 software. An inversion pulse is applied, the spectrometer drives the syringe pump to move the sample a known distance into the evolution field. After the evolution time, the spectrometer triggers the syringe pump to withdraw by a similar amount. 
Figure 2. Simulated field plot for magnet geometry employed in this sensor. Fields are not indicative of final values but the relative magnitudes are correct. The detection system is to the left, the sample is pumped between the magnets.

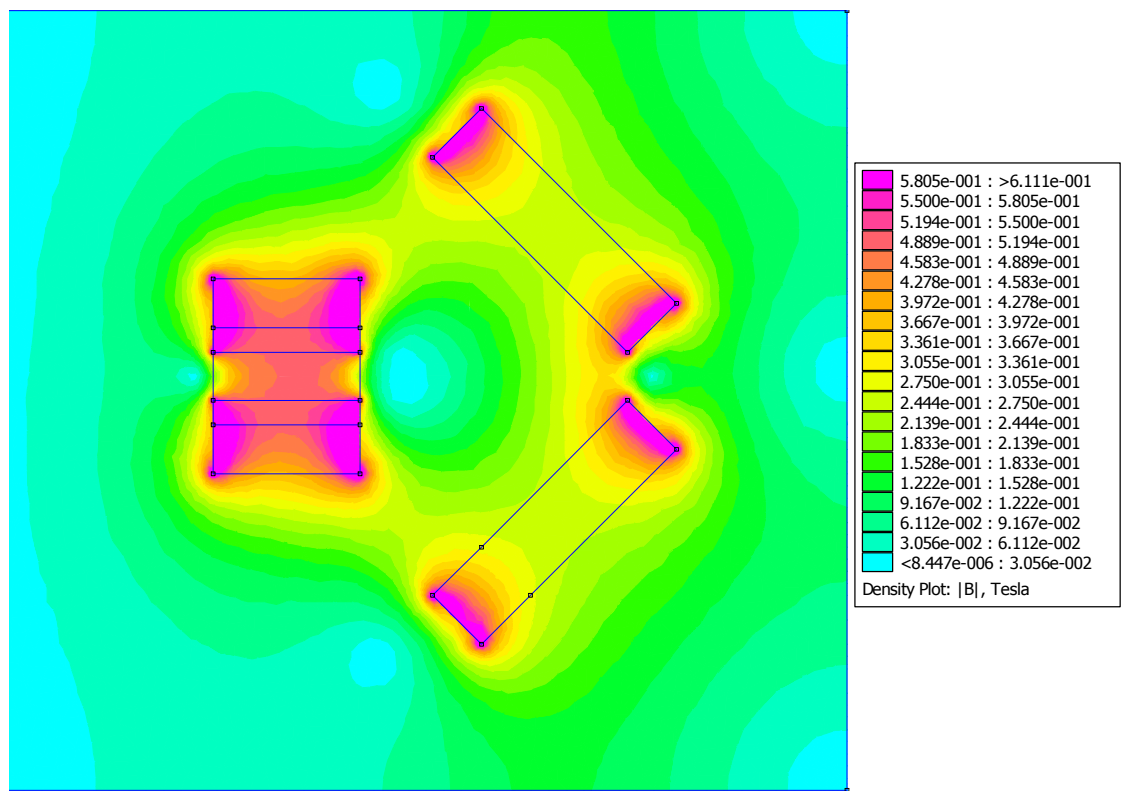

Figure 3. The inversion recovery pulse sequence applied to the sample.

\section{Field}

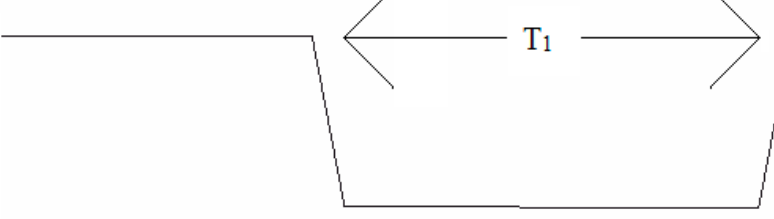

Polarisation

Evolution

Detection

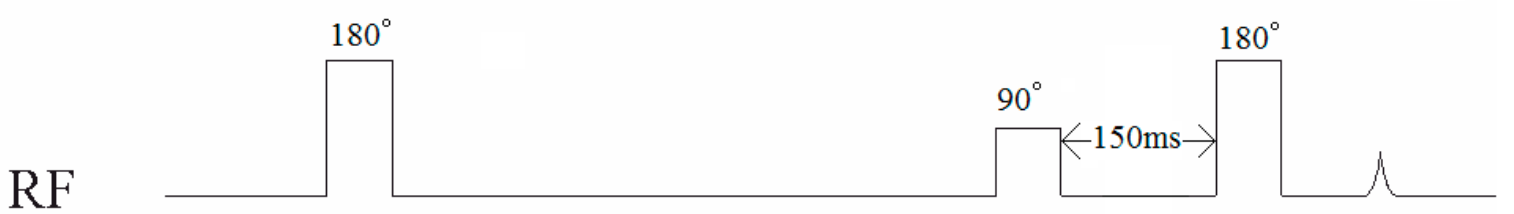

\section{Results and Discussion}

The results (Fig.4) show the change in $T_{1}$ as a result of the change of evolution field strength achieved by changing the infusion and withdrawal volume of the syringe pump. The data is collected by changing the evolution time, which is analogous to the inversion time in a traditional inversion recovery sequence. This is then repeated for different infusion volumes. The blue data in the insert of Figure 4 was moved a long distance from the RF coil and therefore had a relatively high magnetic field acting on it, whilst the data shown in red was propelled a much shorter distance out of the Halbach and evolved within a lower field strength. The gradients of these curves represents $T_{1}$ which is plotted against evolution field strength in Figure 4. 
Figure 4. Plot of spin lattice relaxation time against evolution field strength showing the expected relationship of increasing relaxation time with field strength. Two inversion recovery measurements are shown as an inset for this data. The gradients of these lines represents $\mathrm{T}_{1}$.

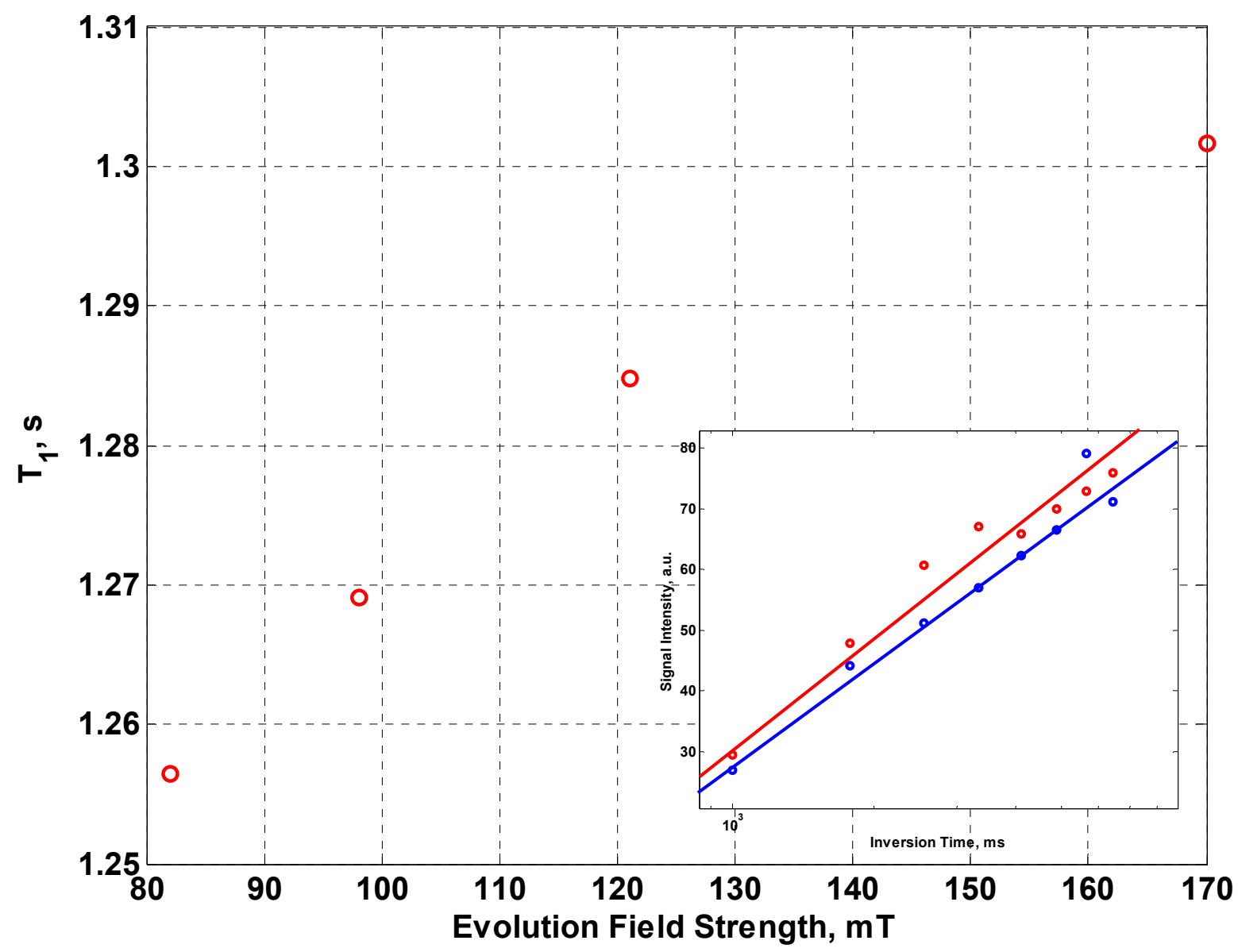

These very preliminary results evidence that this method of propelling a liquid sample between magnetic field strengths is suitable to acquire $T_{1}$ data at different fields and shows great promise for using low cost permanent magnets to produce a portable sensor system for this purpose. As a low cost portable sensor system, this will have numerous applications for example in food process monitoring.

\section{Conclusions}

This preliminary study shows promise for the feasibility of creating a portable permanent magnet fast field cycling system that is low cost. Further development work is required to provide quantitative values for the field strength as a function of position and to minimize the range of fields which are experienced by the samples during the evolution period. The next version of this sensor will use a stepped array of magnets to provide discrete fields over the entire length of the sample.

\section{Acknowledgments}

The authors wish to thank Caroline Shaw for her assistance with this work. 


\section{Author Contributions}

ERD - Prepared manuscript and ran experiments, THR - Designed Halbach magnet.

STP - Constructed Halbach magnet, MIN - Programmed hardware, RHM - Constructed hardware and programmed MR console.

\section{Conflicts of Interest}

The authors declare no conflict of interest.

\section{References}

1. Morris, R.H.; Newton, M.I. Magnetic Resonance Sensors. Sensors 2014, 14, 21722-21725.

2. Morris, R. H.; Newton, M. I.; Knowles, P. R.; Bencsik, M.; Davies, P. A.; Griffin, P.; McHale, G. Analysis of clogging in constructed wetlands using magnetic resonance. Analyst 2011, 136(11), 2283-2286.

3. Kimmich, R.; Field cycling in NMR relaxation spectroscopy: applications in biological, chemical and polymer physics. Bull. Magn. Reson. 1980, 1(4), 195-218.

4. Kimmich, R.; Anoardo, E.; Field-cycling NMR relaxometry, Progress in Nuclear Magnetic Resonance Spectroscopy, Volume 44, Issues 3-4, 30 July 2004, Pages 257-320, ISSN 0079-6565, http://dx.doi.org/10.1016/j.pnmrs.2004.03.002.

5. Meeker, D.C.; Finite Element Method Magnetics, Version 4.2 (15Nov2013 Build), http://www.femm.info

6. Fukushima, E.; Roeder, S.B.W. Relaxation. In Experimental pulse NMR: A nuts and bolts approach; Addison-Wesley Pub., Advanced Book Program.: Reading, Mass., United States, 1981; pp. 168-176.

7. Pine, K. J.; Goldie, F.; Lurie, D. J. In vivo field-cycling relaxometry using an insert coil for magnetic field offset. Magn Reson Med 2014, 72; pp. 1492-1497. doi: 10.1002/mrm.25040

(C) 2015 by the authors; licensee MDPI, Basel, Switzerland. This article is an open access article distributed under the terms and conditions of the Creative Commons Attribution license (http://creativecommons.org/licenses/by/4.0/). 